\title{
Elektronische Zigaretten (E-Zigaretten)
}

Macé M. Schuurmans, Lucrezia Meier-Schatz, Chung-Yol Lee: im Namen der Eidgenössischen Kommission für Tabakprävention (EKTP)*

\section{Einleitung}

Bei der Diskussion um die Stellung der E-Zigarette müssen drei Prinzipien berücksichtigt werden:

1. Das Realitätsprinzip, das bedeutet, dass akzeptiert werden muss, dass E-Zigaretten in der Schweiz bereits erhältlich sind und gebraucht werden, obwohl die Auswirkungen auf die Gesundheit und die Tabakabhängigkeit noch nicht genügend bekannt sind.

2. Das Prinzip der Prävention, das bedeutet, Nichtrauchende, besonders Kinder und Jugendliche, vor einem Konsumprodukt zu schützen, das einen erleichterten Zugang zum Tabakkonsum ermöglicht. Es soll auch verhindert werden, dass Rauchen wieder als normales Verhalten betrachtet wird.

3. Das Prinzip der Vorsicht, das bedeutet, dass Nichtrauchende geschützt werden müssen (Passivkonsum), da die Langzeitwirkungen auf die Gesundheit nicht bekannt sind.

\section{Stand der Forschung}

a. Unter dem Begriff «elektronische Zigaretten» versteht man gegenwärtig eine breite Palette von verschiedenen Produkten. Gemeinsam ist ihnen, dass sie prinzipiell aus folgenden Teilen bestehen: einer Batterie, einem Zerstäuber, einem Reservoir oder einer Kartusche mit der zu verdampfenden Flüssigkeit (Liquid) sowie einem Mundstück. Sie funktionieren entweder auf Druck eines Schalters oder durch Saugen des Benutzers am Mundstück, welches über einen Sensor den Zerstäuber bzw. das Heizelement aktiviert.

b. Die zu verdampfenden Flüssigkeiten (Liquide) in den Kartuschen enthalten eine Mischung aus Propandiol (Propylenglycol), Glycerol und Wasser in unterschiedlichen Konzentrationen sowie aus Aromen. Es gibt diese Flüssigkeiten sowohl mit als auch ohne Nikotin.

c. Gegenwärtig dürfen in der Schweiz nur Liquide verkauft werden, die kein Nikotin enthalten. Kartuschen mit Nikotin können im Ausland oder über das Internet aus dem Ausland bestellt werden. Pro Person sind maximal $150 \mathrm{ml}$ pro Kauf erlaubt [1].

d. Die Benutzer von E-Zigaretten sind hauptsächlich ehemalige oder aktuelle Raucherinnen und Raucher [2] sowie Jugendliche und junge Erwachsene [3]. Die
Lebenszeitprävalenz der Nutzung von E-Zigaretten (mindestens einmalige Anwendung) im Jahr 2015 war in der Schweiz 14\% in der Bevölkerung ab 15 Jahren; dabei wurden E-Zigaretten mit oder ohne Nikotin berücksichtigt [3]. Deutlich erhöhte Anteile weisen Personen unter 35 Jahren (zwischen 24,8 und 33,0\%), Personen in Ausbildung (27,4\%) und die Bevölkerung der Westschweiz (19,6\%) auf [3]. Die Jugendlichen und jungen Erwachsenen sind die häufigsten Anwender von E-Zigaretten, wobei nur ein kleiner Anteil regelmässige (mindestens einmal pro Woche) Anwender sind. Die regelmässige Anwendung von E-Zigaretten wird bei 0,7\% der Bevölkerung angegeben. Als häufigste Gründe für deren Konsum werden genannt: ein Produkt konsumieren, das weniger giftig ist als Tabak; den Tabakkonsum verringern; mit Rauchen aufhören und den Rückfall vermeiden; in Nikotinprodukt konsumieren, das billiger ist als Tabakzigaretten; einen Tabakersatz verwenden an jenen Orten, wo Rauchen verboten ist.

e. Unter den Benutzern von E-Zigaretten sind auch neugierige Jugendliche, sogar Kinder, die gerne etwas Neues ausprobieren wollen.

f. Die kurzfristigen, negativen Folgen für die Gesundheit sind Trockenheit und lokale Irritationen (von Nase und Rachen), Kopfschmerzen, Atembeschwerden, Husten [4]. Wenn unreines Glycerol verwendet wird oder wenn das Liquid durch den Benutzer verändert wird, kann dies zur Folge haben, dass Öl inhaliert wird, was zu einer Lungenentzündung führen kann [5]. Technische Mängel können zum Austritt von nikotinhaltigen Lösungen und zur Aufnahme via Haut oder Schleimhaut führen oder auch eine Überhitzung mit Explosion bewirken mit entsprechenden Verletzungen [6, 7]. Es besteht auch die Gefahr einer akzidentellen Nikotin-Intoxikation durch das Verschlucken von Nachfüllflüssigkeiten besonders bei Kleinkindern.

g. Bei Nichtrauchenden kann der Konsum von E-Zigaretten zu Nikotinabhängigkeit führen. Design und Farbwahl vieler E-Zigaretten-Modelle und die zahlreichen süsslichen Aromen der Liquide sind vorwiegend für Kinder, Jugendliche und junge Erwachsene ansprechend. Es überrascht daher nicht, wenn Kinder und Jugendliche mit E-Zigaretten experimen- 
tieren und dadurch dem erheblichen Risiko einer Nikotinabhängigkeit ausgesetzt werden.

h. Weitere kurzfristige gesundheitliche Auswirkungen sind möglich, wie z.B. Erhöhung des Blutdrucks, Lungen- und/oder Krebserkrankungen durch Aromen und andere chemische Verbindungen wie z.B. Diacyl, Formaldehyd und andere krebserregende Substanzen [5, 8, 9]. Die mittel- und langfristigen Folgen des Konsums sind nicht bekannt.

i. Die E-Zigarette unterscheidet sich von der traditionellen Zigarette gerade dadurch, dass sie keinen Tabak enthält und dass keine Verbrennungsprodukte des Tabaks inhaliert bzw. ausgestossen werden. Herz-Kreislauf-, Krebs- und/oder Lungenkrankheiten sind bei Anwendung von E-Zigaretten aufgrund des aktuellen Wissensstandes wahrscheinlich vermindert, können jedoch nicht ausgeschlossen werden.

j. Zurzeit gibt es nur wenige aussagekräftige (randomisierte) Studien, die den Nutzen von E-Zigaretten als wirksames Entwöhnungsmittel für die Tabakabhängigkeit belegen. Diese ersten Studien wurden unter Fachpersonen kontrovers diskutiert, denn sie können den Wert der E-Zigarette als Entwöhnungsmittel gegenüber registrierten Rauchstopp-Medikamenten nicht belegen [10].

k. Der Konsum von nikotinhaltigen E-Zigaretten ist mit einem hohen Risiko verbunden, nikotinabhängig zu werden. Dieses Risiko muss besonders im Fall jener Jugendlichen beachtet werden, die keinen Tabak rauchen.

1. Der Markt der E-Zigaretten kann eine Marktnische sein für die Tabakindustrie, die ein klares Interesse für die neuen Produkte zeigt, da in vielen westlichen Ländern der Verkauf von Tabakzigaretten spürbar rückläufig ist.

m. Die Schweizer Expertinnen und Experten der Tabakprävention sind geteilter Meinung, was die E-Zigaretten betrifft [11]. Einige sehen in diesem Produkt ein Instrument mit einem Potential, Gesundheitsrisiken zu reduzieren. Andere sehen die Gefahr, dass die E-Zigarette auf individueller Ebene den Einstieg in die Tabakabhängigkeit fördert und auf gesellschaftlicher Ebene das Rauchen wieder normalisiert.

n. Die EKTP hat das Experten-Konsens-Verfahren (Delphi-Methode) früher unterstützt und die Schlussfolgerungen dieser Studie grösstenteils in ihren Empfehlungen übernommen [11]. Neuere Erkenntnisse aus anderen Studien und Untersuchungen wurden bei der Überarbeitung der Stellungnahme ebenfalls berücksichtigt (siehe Kapitel 4).

o. Aufgrund des aktuellen Kenntnisstandes sind E-Zigaretten nach kurzdauernder Anwendung ohne Wiederholung weniger schädlich als Tabakzigaretten. Mangels Daten zu mittel- und langfristigen ge- sundheitlichen Auswirkungen der E-Zigaretten ist momentan eine Einschätzung der relativen Risikoreduktion durch den kompletten Wechsel von Tabakzigaretten auf E-Zigaretten nicht genau festlegbar. Eine entsprechende Einschätzung aus Grossbritannien wird von Expertinnen und Experten kritisch hinterfragt [12].

\section{Empfehlungen}

1. E-Zigaretten müssen einer spezifischen Regulierung unterworfen werden. Dazu gehören Qualitätsnormen für die Spezifikationen der E-Zigaretten-Geräte, die Inhalte der Kartuschen (Liquide), eine restriktive Liste der erlaubten Komponenten dieser Flüssigkeiten sowie eine Höchstgrenze für den Nikotingehalt. Alle Inhaltsstoffe müssen auf den Packungen aufgeführt werden, wo ebenfalls Warnhinweise zu den Risiken beim Gebrauch anzubringen sind.

2. Das Bundesgesetz zum Schutz vor Passivrauchen sowie die ergänzenden kantonalen Gesetzgebungen gelten auch für den Gebrauch von E-Zigaretten.

3. Jede Form von Werbung und Promotion für E-Zigaretten ist verboten. Dies, weil vulnerable Zielgruppen (Kinder und Jugendliche) immer auch angesprochen werden und weil Studienresultate darauf hinweisen, dass der Konsum von E-Zigaretten bei Jugendlichen das Risiko für einen späteren Konsum von Tabakzigaretten erhöht.

4. In Übereinstimmung mit den Empfehlungen der WHO sollen Gesundheitsbehörden davon absehen, EZigaretten für die Tabakentwöhnung zu empfehlen [13]. Die nötigen wissenschaftlichen Nachweise für die Sicherheit und die langfristige Wirksamkeit fehlen für eine solche Empfehlung. Zu empfehlen sind die Behandlungsarten der Tabakabhängigkeit, die ihre Effizienz bereits bewiesen haben (Substitutionsprodukte, Bupropion, Vareniclin, Rauchstopplinie, ärztliche und nichtärztliche Fachberatung etc.).

5. Nikotinhaltige E-Zigaretten und Liquide dürfen in der Schweiz nur an Erwachsene verkauft werden.

6. E-Zigaretten müssen gleich wie Zigaretten besteuert werden. Die Höhe der Steuer soll derjenigen der Tabakprodukte entsprechen. Der Steuerertrag soll dazu verwendet werden, die Tabakprävention sowie die Forschung im Bereich der E-Zigaretten zu fördern. Dabei muss der Fokus auf die Analyse der Langzeitwirkung auf die Gesundheit, der Effizienz für die Tabakentwöhnung, der psychologischen und sozialen Auswirkungen sowie der gleichzeitigen Konsumation von Tabakprodukten und E-Zigaretten gelegt werden.

7. Regelmässige Analysen von häufig verkauften Liquiden sind erforderlich. Zielführend sind koordinierte Untersuchungen unter den Kantonen. 


\section{Literatur}

1 Charrière R. Informationsschreiben Nr. 146: Elektrische Zigaretten, elektronische Zigaretten, E-Zigarette. 2010. BAG, Bern.

2 Etter JF, Bullen C. Electronic cigarette: users profile, utilization, satisfaction and perceived efficacy. Addiction, 2011;106(11):2017-28.

3 Kuendig H, Notari L, Gmel G. Die E-Zigarette in der Schweiz im Jahr 2015 - Auswertung der Daten des Suchtmonitorings Schweiz. 2016. Sucht Schweiz, Lausanne.

4 Caponnetto P, et al. Efficiency and Safety of an eLectronic cigAreTte (ECLAT) as tobacco cigarettes substitute: a prospective 12-month randomized control design study. PLoSONE, 2013;8(6):e66317.

5 Hua M, Talbot P. Potential health effects of electronic cigarettes: A systematic review of case reports. Prev Med Rep. 2016 Jun 10; 4:169-78.

6 Maina G, Castagnoli C, Passini V, Crosera M, Adami G, Mauro M, Filon FL. Transdermal nicotine absorption handling e-cigarette refill liquids. Regul Toxicol Pharmacol. 2016 Feb;74:31-3.

7 Nicoll KJ, Rose AM, Khan MA, Quaba O, Lowrie AG. Thigh burns from exploding e-cigarette lithium ion batteries: First case series. Burns. 2016 Jun;42(4):e42-6.
8 Schuurmans MM. Elektronische Zigaretten und kardiovaskuläres Risiko. Herz+Gefäss 2016;1:6-9.

9 Farsalinos KE, Kistler KA, Gillman G, Voudris V. Evaluation of elec tronic cigarette liquids and aerosol for the presence of selected inhalation toxins. Nicotine Tob Res. 2015 Feb;17(2):168-74.

10 Kaelin R, Barben J, Schuurmans MM. Elektronische Zigaretten, E-Shishas und "Heat but not burn devices». Swiss Med Forum. 2017:17(5) (in print).

11 Blaser J, Cornuz J. Experts' consensus on use of electronic cigarettes: a Delphi survey from Switzerland. BMJ Open. 2015 Apr 15 5(4):e007197.

12 E-cigarettes: Public Health England's evidence-based confusion. Lancet. 2015 Aug 29;386(9996):829.

13 WHO: WHO study group on tobacco product regulation (TobReg) report on the scientific basis of tobacco product regulation: third report of a WHO study group, WHO technical report series no. 955 2009. http://www.who.int/tobacco/global_interaction/tobreg 